[0212-7199 (2006) 23: 6; pp 285-287] ANALES DE MEDICINA INTERNA Copyright $\odot 2006$ ARAN EDICIONES, S.L.

AN. MED. INTERNA (Madrid) Vol. 23, N. ${ }^{\circ} 6$, pp. $285-287,2006$

\title{
Pancarditis neumocócica con infarto agudo de miocardio y fracaso cardiaco de curso fulminante
}

\author{
M. A. GARCÍA ORDÓÑEZ, I. HIERRO MARTÍN ${ }^{1}$, J. J. JIMÉNEZ MARTÍN ${ }^{1}$, \\ J. BLASCO MORILLA ${ }^{2}$ \\ Servicios de Medicina Interna, ${ }^{1}$ Anatomía Patológica $y{ }^{2}$ Cuidados Intensivos. Hospital de \\ Antequera. Málaga
}

\begin{abstract}
RESUMEN
Streptococcus pneumoniae es una causa infrecuente de endocarditis infecciosa en los adultos. Típicamente ocurre en individuos con enfermedades debilitantes como alcoholismo. El curso evolutivo es agresivo, con rápida destrucción valvular, por lo que se asocia a una alta mortalidad. Presentamos el caso de un paciente joven sin enfermedades subyacentes con endocarditis y meningitis neumocócica neumocócica de curso fulminante demostrada en estudio post mórtem.
\end{abstract}

PALABRAS CLAVE: Streptococcus pneumonia. Endocarditis. Infarto de miocardio.
FULMINANT PNEUMOCOCCAL PANCARDITIS AND ACUTE MYOCARDIAL INFARCTION WITH CARDIAC FAILURE

\begin{abstract}
Streptococcus pneumoniae is an infrequent cause of endocarditis in adults. Typically occurs in individuals with debilitating illnesses as alcoholism. Frequently the course is aggressive, with rapid destruction of valves. It is associated with high mortality. We describe a young patient without underlying illnesses with pneumococcal endocarditis and meningitis of fulminant course diagnosed in postmorten examination.
\end{abstract}

KEY WORDS: Streptococcus pneumoniae. Endocarditis. Myocardial infarction.

García Ordóñez MA, Hierro Martín I, Jiménez Martín JJ, Blasco Morilla J. Pancarditis neumocócica con infarto agudo de miocardio y fracaso cardiaco de curso fulminante. An Med Interna (Madrid) 2006; 23: 285-287.

\section{INTRODUCCIÓN}

A pesar de que Streptococcus pneumoniae es un germen ampliamente distribuido, es una causa infrecuente de endocarditis bacteriana, estimándose una frecuencia inferior al 1-3\% de los casos (1). En los últimos años se han publicado casos esporádicos y series pequeñas lo que pudiera indicar un incremento en su frecuencia (2-4). En las últimas décadas ha habido importantes progresos en el diagnóstico ecocardiográfico y en el manejo terapéutico que ha llevado a mejorar el pronóstico de muchas formas de endocarditis. Sin embargo, la endocarditis neumocócica (EN) mantiene una alta tasa de mortalidad, entre $28-60 \%$ (1-4), debido a su curso agresivo y a una rápida destrucción valvular. Presentamos el caso de varón joven con EN que evolucionó de forma fulminante.

\section{CASO APORTADO}

Varón de 20 años que acudió a urgencias por cuadro febril de $39^{\circ} \mathrm{C}$ de 4 días de evolución con escalofríos, disnea moderada, náuseas y vómitos acompañado de dolorimiento abdominal difuso.

El paciente había estado sano sin padecer enfermedades previas de interés. No tenía contactos con animales, ni había consumido lácteos sin higienizar. No había sufrido manipulación dentaria ni otro tipo de instrumentalización reciente.

La exploración a la admisión fue: tensión arterial de 90/50 $\mathrm{mmHg}$, la temperatura fue $38,5^{\circ} \mathrm{C}$. Estaba alerta, orientado, con buen estado general, bien perfundido, normocoloreado. Presentaba ligera taquipnea. No presentaba ingurgitación venosa yugular y las carótidas latían simétricas. A la auscultación el corazón latía rítmico, con un soplo protosistólico II-III/VI en foco aórtico. El murmullo vesicular estaba conservado. A la palpación abdominal había dolorimiento difuso sin semiología de peritonismo, sin masas ni organomegalias. El peristaltismo intestinal estaba conservado. No había signos de afectación o focalidad neurológica. Los pulsos periféricos estaban presentes. No se apreciaban lesiones petequiales ni otras lesiones dérmicas.

Los test de laboratorio mostraban leucocitosis de $13.900 / \mathrm{mm}^{3}$ con desviación a la izquierda y trombopenia marcada de 54.600 $/ \mathrm{mm}^{3}$. La serie roja y los test plasmáticos de coagulación eran normales. El frotis de sangre periférica mostró leucocitosis con marcada

Trabajo aceptado: 20 de diciembre de 2005

Correspondencia: Miguel Ángel García Ordóñez. Avda. Juan Sebastián Elcano, 46, ático C. 29007 Málaga. Fax: 952844411. e-mail: magomalaga@ vodafone.es 
granulación de los leucocitos. La urea fue $39 \mathrm{mg} / \mathrm{dl}$ y la creatinina $1,4 \mathrm{mg} / \mathrm{dl}$. Los electrolitos eran normales. La radiografía de tórax fue normal. Se obtuvieron hemocultivos y se inició tratamiento antibiótico empírico con levofloxacino, ingresando en planta.

En las horas siguientes el paciente presentó deterioro brusco con taquipnea intensa, signos de hipoperfusión periférica, crepitantes finos diseminados a la auscultación con tonos cardiacos taquiarritmicos e hipotensión arterial severa, sin respuesta a aporte de expansores de volumen. Se trasladó a la Unidad de Cuidados Intensivos. Una radiografía de tórax urgente mostró signos de fracaso cardiaco. El paciente sufrió parada cardiorrespiratoria. A pesar de las maniobras de resucitación cardiopulmonar avanzadas con intubación orotraqueal y conexión a ventilación mecánica, aporte de fluidos y drogas vasoactivas, el paciente falleció.

En el examen post morten, los hallazgos macroscópicos más relevantes fueron: en la cavidad craneal, la pérdida de transparencia de las meninges, de aspecto congestivo y edematoso; en el tórax se observó líquido hemorrágico tanto a nivel de espacio pleural como pericárdico que se tomó muestra para cultivo; en el corazón la válvula aórtica presentaba destrucción de dos de sus tres valvas (Fig. 1); el tejido miocárdico en el cono de salida del ventrículo izquierdo, a nivel de la arteria coronaria izquierda, y extendiéndose hasta el septum, estaba necrótico; la cavidad abdominal presentaba líquido ascítico claramente hemorrágico; el hígado y el intestino presentaban congestión vascular; el bazo era de aspecto hemorrágico, con zonas de necrosis y rotura capsular.

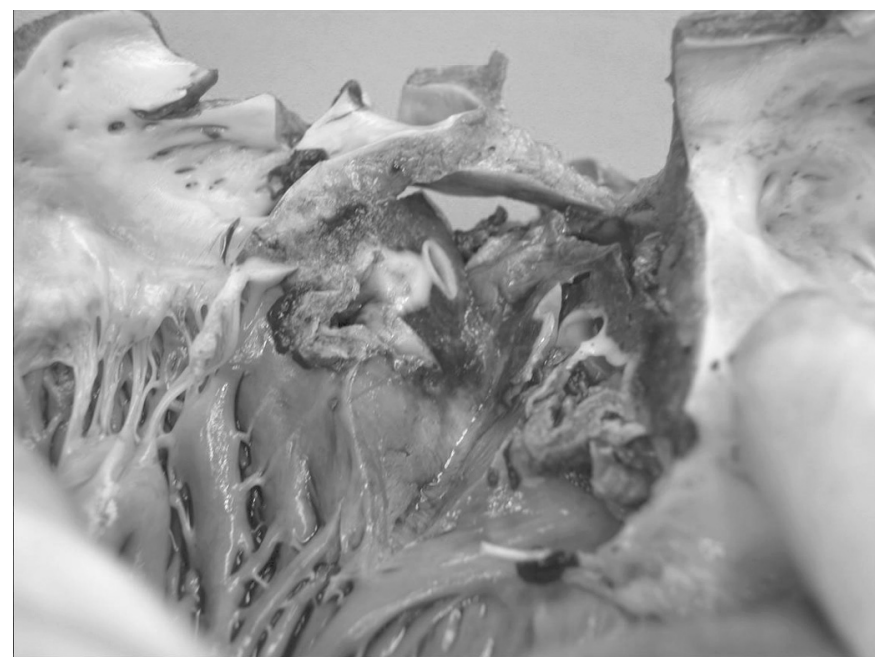

Fig. 1. Válvula aórtica con destrucción de dos de sus tres valvas.

Microscópicamente, la válvula aórtica destruida tenía en sus valvas acúmulos de fibrina en forma de vegetaciones, con múltiples imágenes de microorganismos en su interior; se observaba intenso infiltrado inflamatorio polimorfonuclear desde endocardio a pericardio, en forma de absceso perivalvular, que afectaba intensamente la salida de la arteria coronaria izquierda, y que estaba rodeada de extensas áreas de necrosis isquémica miocárdica (Fig. 2). Posteriormente se recibió la serología para el VIH que fue negativa. Tanto en el hemocultivo previo como el cultivo postmorten de líquido pericárdico creció streptococcus pneumoniae serotipo $18 \mathrm{C}$, sensible a penicilina. Los cultivos de líquido pleural y ascítico fueron negativos.

\section{DISCUSIÓN}

Los factores predisponentes para EN no están claramente definidos, aunque probablemente sean muy similares a los que presentan los individuos con enfermedad neumocócica invasiva en general. Tales factores incluyen condiciones que afectan los mecanismos de aclaración local (tabaquismo,

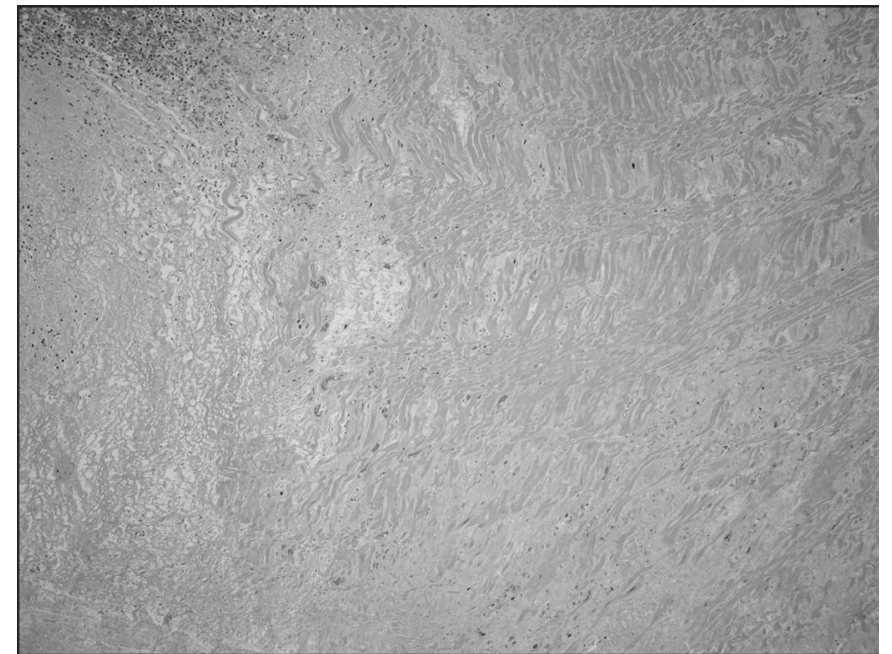

Fig. 2. Corte histológico a nivel perivalvular que muestra infiltrado inflamatorio rodeado de extensa zona de necrosis.

enfermedad pulmonar crónica) y condiciones caracterizadas por una respuesta inmune del huésped alterada (edades extremas de la vida, alcoholismo, diabetes mellitus, cirrosis hepática, insuficiencia renal crónica y todas aquellas situaciones que conllevan un cierto grado de inmunodeficiencia) (5). No obstante, en un 22-33\% de los casos no se describen factores predisponentes (6-8). La duración media de la enfermedad antes del diagnóstico varía entre 7-9 días, siendo en muchos casos el diagnóstico postmorten (12\%) (5).

La puerta de entrada pulmonar es, con mucho, la más frecuentemente descrita. La EN se asocia concomitantemente a neumonía en $35-60 \%$ casos y meningitis en $29-60 \%$ casos (58). La válvula aórtica es la más frecuentemente afectada en la EN (45-74\%) (5-7). En el 66-84\% de los casos de EN no hay cardiopatía estructural subyacente (8).

El curso de la EN es típicamente agresivo, asociándose con unas altas tasas de morbilidad y mortalidad. Así, en la presentación clínica es característico que falten los estigmas periféricos propios de la endocarditis infecciosa (solo presentes en el $11 \%$ de los casos), lo cual puede retrasar el diagnóstico (5). Por otro lado, la capacidad del Streptococcus pneumoniae de atacar válvulas cardiacas normales, con rápida destrucción del tejido endotelial, principalmente de la válvula aórtica, provoca la frecuente aparición de complicaciones severas como fallo cardiaco congestivo (49-69\%), embolización (24-38\%) y shock (3-13\%) (5-8).

Aunque no existen datos sobre la terapia antibiótica empírica óptima para pacientes con EN, en base a la naturaleza agresiva de esta enfermedad así como a la frecuente asociación con meningitis, una pauta recomendable, mientras se conocen los patrones de sensibilidad, sería aquella que se realiza en los casos que se sospecha meningitis neumocócica (ceftriaxona más vancomicina) (5). El impacto de la emergente resistencia del Streptococcus pneumoniae a la penicilina sobre la presentación clínica y pronóstico de la EN no ha sido claramente evaluada. Estudios recientes han descrito tasas de resistencia a penicilina en la EN similar a la que presenta la enfermedad neumocócica invasiva (17-38\%) sin que se asocie a un peor pronóstico de la EN.7-8 El tratamiento medico-quirúrgico con antibioterapia combinada y reemplazamiento valvular precoz ha mostrado una significativa disminución de la mortalidad $(5,8)$. 


\section{Bibliografía}

1. Bayliss R, Clarke C, Oakley CM, Somerville W, Whitfield AGW, Young SEJ. The microbiology and pathogenesis of infective endocarditis. $\mathrm{Br}$ Heart J 1983; 50: 513-9.

2. Powderly WG, Stanley SL, Medoff G. Pneumococcal endocarditis: report of a series and review of the literature. Rev Infect Dis 1986; 8: 786-91.

3. Ugolini V, Pacifico A, Smitherman TC, Mackowiak PA. Pneumococcal endocarditis update: analysis of 10 cases diagnosed between 1974 and 1984. Am Heart J 1986; 112: 813-8.

4. Bruyn GW, Thompson J, Van Der Meer JWM. Pneumococcal endocarditis in adult patients. A report of five cases and review of the literature. Q J Med 1990; 74:33-40.

5. Aronin SI, Mukherjee SK, West JC, Cooney EL. Review of pneumococ- cal endocarditis in adults in the penicillin era. Clin Infect Dis 1998; 26: 165-71.

6. Lindberg J, Prag J, Schonheyder HC. Pneumococcal endocarditis is not just a disease of the past: An analysis of 16 cases diagnosed in Denmark 1986-1997. Scand J Infect Dis 1998; 30: 469-72.

7. Martínez E, Miro JM, Almirante B, Aguado JM, Fernández-Viladrich P, Fernández-Guerrero ML, et al. Effect of penicilin resistente of Streptococcus pneumoniae on the presentation, prognosis, and treatment of pneumococcal endocarditis in adults. Clin Infect Dis 2002; 35: $130-9$.

8. Lefort A, Mainardi JL, Selton-Suty C, Casassus P, Guillevin L, Lortholary O, et al. Streptococcus pneumoniae endocarditis in adults: a multicenter study in France in the era of penicillin resistance (1991-1998). 